\title{
Preoperative assessment of prognostic factors in breast cancer
}

\author{
H Denley, S E Pinder, C W Elston, A H S Lee, I O Ellis
}

\begin{abstract}
The adoption of preoperative diagnostic strategies involving fine needle aspiration cytology (FNAC) or core biopsy is well established, allowing the planning of operating lists and bed occupancy and patient involvement in therapeutic management. In addition to diagnosis, however, pathologists are increasingly being asked to provide pathological prognostic information from preoperative samples. This leader describes techniques for predicting prognosis and response to treatment on these specimens and some of the problems inherent in the determination of prognosis on small samples. For example, although histological grade can be assessed relatively reliably on either core or FNAC samples, the evaluation of tumour type (which includes an overall assessment of the architecture of a given tumour) may be less reliable on small preoperative samples. Other well recognised histological prognostic factors, such as vascular channel invasion or tumour size, cannot be determined accurately on small preoperative samples. For those patients who might benefit from neoadjuvant treatment, predicting the response to such treatments-for example, by the assessment of oestrogen receptor status-can readily be performed on either core biopsy or FNAC. In the future, other molecular markers such as C-erbB-2 might also prove beneficial in predicting response to newly developed treatments. (F Clin Pathol 2001;54:20-24)
\end{abstract}

Keywords: breast cancer; fine needle aspiration cytology; needle core biopsy; tumour grade

Any case of suspected carcinoma of the breast should be evaluated initially with a triple assessment comprising clinical, radiological, and pathological review. ${ }^{1-3}$ Pathological assessment will involve the evaluation of either a fine needle aspiration cytology (FNAC) sample or needle core biopsy (NCB) specimen. The relative frequency of utilisation of these procedures has varied over the past three decades and, in part, depends on local and personal preferences.

FNAC is a technique that has been used for over a century. ${ }^{45}$ It is relatively inexpensive and can be performed either freehand or with image guidance (ultrasound or stereotactic). However, it requires regular practice to ensure the maintenance of aspirator skill ${ }^{7}$ and may cause difficulty in interpretation for the less experienced pathologist. Thus, training and experience are required in both the aspirator and the pathologist. Air dried or fixed smears, cytospin, or cytoblock preparations can be used. In addition, a variety of staining methods can be used, including Giemsa, haematoxylin and eosin ( $\mathrm{H}$ and $\mathrm{E})$, Papanicolaou, DiffQuick, and periodic acid Schiff (PAS). In the right hands, the procedure is both sensitive (range, 74-96\%) and specific (with most series approaching $100 \%),{ }^{8-10}$ thereby fulfilling the minimum requirements of the National Health Service Breast Screening Programme (NHSBSP) ${ }^{11}$ The main drawback with FNAC, however, is the inability to provide morphological information, making the distinction between in situ and invasive carcinoma impossible at the preoperative stage. Therefore, it may not help in the assessment of the requirement for axillary lymph node dissection (unless a corresponding FNAC of an axillary lymph node reveals metastatic carcinoma).

NCB can also be performed either freehand or with image guidance. The technique can be undertaken by surgeons or radiologists and has more reliable and reproducible results. ${ }^{12} \mathrm{NCB}$ specimens can also be evaluated easily by histopathologists to whom $\mathrm{H}$ and $\mathrm{E}$ stained sections are familiar. ${ }^{13}$ However, the patient needs to be given a local anaesthetic, because the needle is larger than that used in FNAC, although the procedure is less traumatic with the newer automated "gun" devices. Even with little experience, good adequacy rates can be achieved, although this does, in part, depend on the number of cores taken. ${ }^{14}$ It has been shown to be more sensitive $(97 \%)$ and specific $(100 \%)$ than FNAC, $^{15}$ and provides useful morphological information, particularly with respect to the presence of invasion in the sample. The presence of screen detected microcalcification may be confirmed and assessed in NCB samples and, in some instances, this can obviate the need for unnecessary surgery when a benign diagnosis is made. For these reasons, many centres have now switched from FNAC to NCB. However, it has been shown that the use of both techniques together provides the best results. ${ }^{16}{ }^{17}$ The ultimate choice of method used varies according to local and personal preference.

Pathological prognostic factors are used in clinical practice for a variety of reasons. They provide detailed information on the prognosis of an individual patient as a basis for counselling and treatment and may allow stratification of patients according to the treatment regimens that can be used. ${ }^{18}$ They allow the comparison of treatments between groups of patients with similar prognoses and help to
Accepted for publication 25 May 2000 
develop our understanding of breast cancer biology, which may permit the development of new treatment modalities. Predictive, as opposed to prognostic, factors can be identified that assist in the identification of an individual or group of patients who would respond to a particular treatment modality (for example, oestrogen receptor positive tumours).

There is an exhaustive selection of putative prognostic factors available for assessment in breast cancer. ${ }^{19}{ }^{20}$ Some of these may be classified as time dependent or chronological (for example, tumour size, stage, and vascular or lymphatic invasion), whereas the remainder are innate biological features of the tumour. The latter group is extensive and includes histological type and grade, markers of proliferation, tumour DNA content, hormone and growth factor receptors, oncogene/tumour suppressor gene expression, proteases, and second messenger system activity. There is no general consensus as to which of these features should routinely be reported in excision biopsies but tumour grade, lymph node stage, and tumour size have all been shown to be independent prognostic factors in multivariate analysis. These factors, therefore, have been combined to produce the Nottingham prognostic index score. ${ }^{21-23}$ Each patient's tumour has an individual value, which allows the prediction of individual prognosis and decisions can thus be made about appropriate treatment. Other prognostic factors that are routinely recorded within our own practice are tumour type, lymphovascular channel invasion, and oestrogen receptor status.

There are important potential benefits to the identification of prognostic and predictive factors preoperatively on NCB or FNAC. For example, prognostic factor assessment in NCB and FNAC would allow the identification of patients who would benefit from neoadjuvant treatment (predominantly patients with grade 3 tumours); those in whom conservation surgery is inadvisable (patients with definite vascular channel invasion); and those who may benefit from specific treatment modalities (for example, tamoxifen, herceptin). Preoperative assessment, particularly NCB, may be useful in establishing "baseline" information before treatment. In addition, sequential biopsy and radiological assessment may determine the baseline and subsequent response to nonoperative management in those patients who are unfit for surgery.

The value of the assessment of prognostic factors in preoperative samples is in part compromised by problems of sampling error: only a small portion of what might be a heterogeneous tumour is obtained and this may not be representative of the tumour as a whole. This might be particularly true for NCB unless multiple samples are taken. Studies have nevertheless shown that grading and typing of invasive carcinoma on NCB and grading on FNAC correlate well with excision specimens (G McKee, et al. British Society of Clinical Cytology, 1991) $)^{24-32}$ and thus the assessment of prognostic factors on preoperative samples might not be as unreliable as believed previously.

\section{Evaluation of traditional prognostic factors}

TUMOUR GRADE

The grade of an invasive carcinoma of the breast is calculated according to the Nottingham modification of the Bloom and Richardson method. ${ }^{33}$ Tubule formation, nuclear pleomorphism, and mitotic frequency are evaluated. Each variable is scored $1-3$ and the cumulative score used to assign a numerical grade of 1,2, or 3 (see UK NHSBSP reporting guidelines $^{34}$ for more information). The grade derived by this method has been shown in multivariate analysis to be an independent factor in predicting patient prognosis and correlates well with 10 year survival figures. ${ }^{33}$ It has been shown in many series to be reproducible when strict criteria are applied to well fixed specimens. $^{35-37}$

The application of this method to NCB samples appears straightforward because formalin fixation is likely to be optimum. However, as mentioned previously, even if multiple cores are obtained, problems may arise with sampling only a small proportion of a heterogeneous tumour. Tubule formation may therefore be overestimated or underestimated and pleomorphism may be underscored. Mitotic counts might be inaccurate because in tumour grading the periphery of the tumour is assessed-the growing edge of the tumourwhere mitotic figures are more frequent. In addition, the core might have an insufficient amount of tumour to allow 10 high power fields to be counted. As a result, the estimation of mitotic frequency might be inaccurate and is generally underscored in the core biopsy sample. $^{2426}$

Studies have shown ${ }^{24} 2638$ that when estimating histological grade, there is a tendency to underscore rather than overscore on core biopsy samples compared with excision specimens. This might result in some patients who would benefit from receiving neoadjuvant treatment (either chemotherapy or radiotherapy) for grade 3 tumours being excluded, but very few would be given unnecessary treatment. This tendency for undergrading has also been documented in prostatic carcinomas, ${ }^{39} 40$ although in these tumours grading is based mainly on architectural features, rather than nuclear pleomorphism and mitotic frequency.

A variety of techniques has been described for grading breast carcinomas in FNAC samples (G McKee, et al. British Society of Clinical Cytology, 1991)..$^{27-32}{ }^{41}$ Some use fixed smear preparations, ${ }^{32}{ }^{41}$ whereas others can be performed on air dried, Giemsa stained smears. ${ }^{27}$ These methods assess a variety of cytological features, including cell dissociation, nuclear size, cell uniformity, nucleoli, nuclear margin, and nuclear chromatin pattern. Indeed, some groups have confirmed the value of these features individually by morphometry. ${ }^{42}$ Tumours can then be grouped into either high or low grades ${ }^{27}$ or grades 1 to $3^{32}$ accordingly. 
These values have been shown to correlate well with histological grade on excision specimens. In addition, with the McKee system ${ }^{32}$ of cytological grading, most lobular carcinomas (excluding pleomorphic lobular tumours) will be classified as grade 1, whereas on histology these are predominantly, although not invariably, grade 2 tumours, scoring 3 for tubules, 2 for pleomorphism, and 1 for mitotic counting. Although these results do compare with grade on histology, their routine use in preoperative FNAC specimens is not currently advocated in the UK NHSBSP. ${ }^{11}$ It should also be noted that it is not possible to discriminate between in situ and invasive disease on cytology and the grade described may be given to a case of pure ductal carcinoma in situ.

\section{TUMOUR TYPE}

Invasive carcinomas are separated on morphological grounds into one of a variety of specialised types, mixed type, or ductal/no special type (NST). ${ }^{35}$ Special types include tubular, cribriform, mucinous, lobular, and medullary. Further subtyping can be performed and infiltrating lobular carcinoma may be classified as being a classic, alveolar, solid, or tubulolobular variant. Mixed forms including tubular mixed, mixed lobular, NST, or special type plus NST can be identified. Tumour type has been shown to correlate with long term survival, ${ }^{43-45}$ but is of less prognostic importance than grade. ${ }^{46}$ This may be because type and grade show complex inter-relations (for example, tubular carcinomas are by definition grade 1 and medullary carcinomas invariably grade 3 ).

Histological typing of invasive breast tumours is carried out on well fixed samples and involves the assessment of several blocks to determine all of the features present. The evaluation of a variety of features including the extent of tubule formation, the presence of lymphoid stroma, mucin production, syncytial or discohesive growth pattern, and "Indian file" formation allows tumours to be separated according to type. Some groups have suggested that the presence of an invasive lobular component might guide the surgeon to expand the area of excision, because the clinical assessment of the overall extent of tumour invasion is difficult and incomplete removal is more likely. ${ }^{47}$

The evaluation of tumour type is fraught with the same difficulties as grade assessment on NCB. This again stems from the possible problem of obtaining a sample that is not representative of the tumour as a whole. However, certain types can be recognised on NCB (such as mucinous or lobular), although the possibility of a mixed tumour cannot be ruled out. A common problem with attempting to ascribe tumour type on NCB is the confusion between tubular mixed (or tubular variant) carcinomas and invasive NST carcinoma. ${ }^{24}$ This is a result of sampling error and tumour heterogeneity, because tubular mixed carcinomas show a central elastotic area with tubule formation, surrounded by an area resembling invasive NST carcinoma.
It is not possible to confirm the presence of invasive carcinoma on FNAC, so the accurate classification of tumour type is also impossible. However, some information may be obtained to give an indication of type-for example, a mucinous background might suggest a mucinous phenotype, whereas intracytoplasmic mucin or lumina or Indian file rows of cells favour lobular carcinoma. ${ }^{27}$

\section{TUMOUR SIZE}

This is a time dependent prognostic factor, with smaller predicting increased survival. ${ }^{21}{ }^{48-51}$ Although on NCB an evaluation of the minimum size of the tumour may be made, neither FNAC nor NCB is of practical day to day use in the measurement of this parameter. Clinical preoperative assessment is also of limited value, with poor correlation between clinical assessment and the size of the excised tumour. If a preoperative estimate of size is required, this should be performed ultrasonographically. ${ }^{52}$

VASCULAR CHANNEL INVASION

Vascular invasion can be assessed on NCB and may, in association with high grade, indicate patients who require postoperative adjuvant treatment. However, although the presence of definite or probable vascular channel invasion is specific (when identified on NCB, this is confirmed on excision sampling), it is not sensitive (not all cases with vascular invasion on histology are identified on NCB). ${ }^{24}{ }^{38}$ The presence of histologically confirmed vascular channel invasion is associated with an increased risk of metastatic disease, increased risk of local recurrence, and reduced survival..$^{53}$ FNAC cannot be used to determine vascular invasion in invasive breast carcinoma.

LYMPH NODE STAGE

Unless samples (either NCB or FNAC) are also taken from axillary lymph nodes at the time of preoperative assessment, the presence of nodal metastases cannot be predicted preoperatively.

\section{HORMONE RECEPTORS}

Oestrogen receptors (ER) and progesterone receptors (PR) are nuclear steroid hormone receptors. Their activation is linked with the regulation of certain genes, particularly those controlling cell growth. The ER status of a tumour correlates well with patient response to antioestrogen treatments, particularly tamoxifen, but also to gonadotrophin releasing hormone analogues. ${ }^{54-56}$ Therefore, it can be used to identify those patients who might benefit from neoadjuvant endocrine treatment. The combination of the assessment of ER and PR may further refine the prediction of the response to endocrine treatment. However, the ER status does not have independent prognostic value because of its close relation with tumour grade. Thus, ER is best considered as a "predictive" rather than prognostic factor.

On excision samples, ER status is now routinely calculated after immunohistochemical labelling of the receptor. This may be 
performed on formalin fixed, paraffin wax embedded tissue, using an avidin-biotin complex-peroxidase labelling technique. $\mathrm{Nu}$ merical scores can be obtained by assessment of the intensity of staining and the percentage of tumour cells stained, ${ }^{57}$ or by a semiquantitative estimate of only the proportion of nuclei expressing the receptor. ${ }^{58}$ The same technique can be applied to NCB specimens and may also be used for PR status. However, it is important to remember that these are labile soluble antigens and appropriate specimen preparation is vital. The main drawback with their evaluation on preoperative samples is again that of tumour heterogeneity.

ER status assessment of FNAC samples can be carried out using a similar technique to that described for NCB, although different antibodies might be required. However, it is important to be certain that only tumour nuclei are scored, because some normal breast epithelial cells are ER and PR positive, whereas other stromal cells are negative. The inadvertent inclusion of these nuclei in scoring methods will falsely affect the overall score. The assessment of hormone receptors on cytology also requires an additional slide or a cytoblock to be prepared in advance.

\section{MOLECULAR MARKERS}

Apart from the hormone receptors outlined above, a wide range of other molecular markers has been identified in breast cancer. Many of these are of disputed independent prognostic relevance and the list is too exhaustive to make the assessment of these practicable in everyday reporting. Most are associated with mechanisms of differentiation, cell growth, and replication or with vascular invasion and metastatic potential. These markers include epithelial mucins (MUC1/EMA), ${ }^{59}{ }^{60}$ tumour suppressor genes (p53, retinoblastoma gene), ${ }^{61-63}$ oncogenes (c-myc, c-erbB-2, ras), ${ }^{64-66}$ proteases (cathepsin D), ${ }^{67} 68$ growth factor receptors (epidermal growth factor receptor), ${ }^{6169}$ proliferation markers (ki-67/Mib1), ${ }^{68}$ adhesion molecules (E-cadherin, integrins, catenins), ${ }^{71}$ and tumour DNA ploidy and S-phase fraction. ${ }^{72}{ }^{73}$ Many can be assessed by immunohistochemical methods, but the amount of tumour sample required makes their evaluation on small preoperative samples impractical. In addition, although many are of confirmed prognostic value in univariate analysis, their main role is to add information to those factors that reach independent significance (see above), or as research tools in the attempt to understand tumour biology and the development of new treatments.

Early results of antimonoclonal antibody treatments with the novel agent Herceptin show promising results. Therefore, the assessment of cerbB2 (Her2/neu) on NCBs might be useful to identify those patients with tumours suitable for inclusion in therapeutic trials. This may be performed by the determination of the oncoprotein by immunohistochemistry or the identification of gene amplification by fluorescent in situ hybridisation.

\section{Conclusion}

The evaluation of pathological prognostic factors in preoperative breast samples is a worthwhile activity, providing useful information on the presence of invasion (NCB) and tumour grade (NCB and FNAC). It occasionally provides information on vascular channel invasion (NCB) and also some clues as to tumour type (NCB and FNAC). More usefully, hormone receptors may be evaluated. However, as in any other field in histopathology, the evaluation of all of these features requires regular practice and audit of the techniques and methodologies used, with respect to sampling (smear, cytospin, or cytoblock preparation), staining, and pathological interpretation. The use of some of these techniques allows the identification of patients who might benefit from particular treatment modalities and may provide useful information regarding individual patient prognosis.

1 Pinder SE, Elston CW, Ellis IO. The role of pre-operative diagnosis in breast cancer. Histopathology 1996;28:563-6.

2 Lamb J, Anderson TJ, Dixon MJ, et al. Role of fine needle aspiration cytology in breast cancer screening. F Clin Pathol 1987;40:705-9.

3 National Co-ordinating Group for Breast Screening Pathology. Guidelines for pathology services, Publication No. 2. Sheffield: National Co-ordinating Group for Breast
Screening Pathology, 1997.

4 Spriggs AI. History of cytodiagnosis. $\mathcal{F}$ Clin Pathol 1977;30: 1091-102.

5 Frable WJ. Needle aspiration biopsy: past, present and future [review]. Hum Pathol 1989;20:504-17.

6 Snead DRJ, Vryenhoef P, Pinder SE, et al. Routine audit of breast fine needle aspiration (FNA) cytology specimens breast fine needle aspiration (FNA) cytology specimens
and aspirator inadequate rates. Cytopathology 1997;8:236and

7 Lee KR, Foster RS, Papillo JL. Fine needle aspiration of the breast. Importance of the aspirator. Acta Cytol 1987;31: $281-4$

8 Watson DP, McGuire M, Nicholson F, et al. Aspiration cytology and its relevance to the diagnosis of solid tumours of the breast. Surg Gynecol Obstet 1987;165:435-41.

9 Langmuir VK, Cramer SF, Hood ME. Fine needle aspiration cytology in the management of palpable benign and malignant breast disease. Correlation with clinical and mammographic findings. Acta Cytol 1989;33:93-8.

10 Negri S, Bonetti F, Capitanio A, et al. Preoperative diagnostic accuracy of fine needle aspiration in the management of breast lesions: comparison of specificity and sensitivity with clinical examination, mammography, echography and ther-

mography in 249 patients. Diagn Cytopathol 1994;11:4-8.

1 Cytology Sub-group of the National Co-ordinating Committee for Breast Screening Pathology. Guidelines for cytology procedures and reporting in breast cancer screening,
Publication No. 22. Sheffield: National Co-ordinating Publication No. 22. Sheffield: National Co-ordin
Committee for Breast Screening Pathology, 1992.

12 Parker SH, Burbank F, Jackman RJ, et al. Percutaneous large-core breast biopsy: a multi-institutional study. Radiology 1994;193:359-64.

13 Dahlstrom JE, Jain S, Sutton T, et al. Diagnostic accuracy of stereotaxic core biopsy in a mammography breast cancer screening programme. Histopathology 1996;28:421-7.

14 Liberman L, Dershaw DD, Rosen PP, et al. Stereotaxic 14-gauge breast biopsy: how many core biopsy specimens are needed? Radiology 1994;192:793-5.

15 Cheung PS, Yan KW, Alagaratnam TT. The complementary role of fine needle aspiration cytology and tru-cut needle biopsy in the management of breast masses. Aust $N Z \mathcal{F}$ Surg 1987;57:615-20.

16 Litherland JC, Evans AJ, Wilson ARM, et al. The impact of core biopsy on pre-operative diagnosis rate of screen core biopsy on pre-operative diagnosis rate
detected cancers. Clin Radiol 1996;51:562-5.

17 Poole GH, Willsher PC, Pinder SE, et al. Diagnosis of breast cancer with core-biopsy and fine needle aspiration cytology. Aust N Z F Surg 1996;66:592-4.

18 Clark GM. Do we really need prognostic factors for breast cancer? Breast Cancer Res Treat 1994;30:117-26.

19 Elston CW, Ellis IO, Pinder SE. Pathological prognostic factors in breast cancer. Crit Rev Oncol Hematol 1999;31: 209-23.

20 Miller RW, Ellis IO, Sainsbury JRC, et al. ABC of breast diseases-prognostic factors. BMF 1994;309:1573-6.

21 Galea MM, Blamey RW, Elston CW, et al. The Nottingham prognostic index in primary breast cancer. Breast Cancer
Res Treat 1992;22:207-19.

22 Haybittle JL, Blamey RW, Elston CW, et al. A prognostic index in primary breast cancer. Br f Cancer 1982;45:361-6.

23 Todd JH, Dowle C, Williams MR, et al. Confirmation of a prognostic index in primary breast cancer. $B r \mathcal{F}$ Cancer
1987;56:489-92. 
24 Denley H, Ellis IO, Elston CW, et al. An audit of grading and typing of invasive breast carcinoma on needlecore biopsy

25 Dahlstrom JE, Sutton S, Jain S. Histological precision of stereotactic core biopsy in diagnosis of malignant and premalignant breast lesions. Histopathology 1996;28:537-41.

26 Astall EC, Bobrow LG. Assessment of the accuracy of diagnostic and prognostic information provided by core biopsy in breast carcinoma. F Pathol 1998;186:ssPA6.

27 Hunt CM, Ellis IO, Elston CW, et al. Cytological grading of breast carcinoma - a feasible proposition? Cytopathology 1990;1:287-95

28 Sneige N. Nuclear grading in fine needle aspirates of the breast. Cytopathology Annual 1992;2:161-71.

29 Dabbs DJ. Role of nuclear grading of breast carcinoma in fine needle aspirate specimens. Acta Cytol 1993;37:361-6.

30 Dabbs DJ, Silvermann JF. Prognostic factors from the fine needle aspirate: breast carcinoma nuclear grade. Diagn Cytopathol 1994;10:203-8.

31 Ciatto S, Bonardi R, Herd-Smith, et al. Prognostic value of breast carcinoma cytological grading. A retrospective study of 213 cases. Diagn Cytopathol 1993;9:160-3.

32 Robinson IA, McKee G, Nicholson A, et al. Prognostic value of cytological grading of fine-needle aspirates from breast carcinomas. Lancet 1994:343:947-9.

33 Elston CW, Ellis IO. Pathological prognostic factors in breast cancer. I. The value of histological grade in breas cancer: experience from a large study with long term follow up. Histopathology 1991;19:403-10

34 National Co-ordinating Group for Breast Screening Pathology. Guidelines for pathology reporting in breast cancer screening, Publication No. 3, 2nd ed. Sheffield: National Co-ordinating Group for Breast Screening Pathology, 1997.

35 Dalton LW, Page DL, Dupont WD. Histologic grading of breast carcinoma: a reproducibility study. Cancer 1994;73: $2765-70$.

36 Frierson HF, Wolber RA, Berean KW, et al. Interobserver reproducibility of the Nottingham modification of the Bloom and Richardson histological grading scheme for infiltrating ductal carcinoma. Am 7 Clin Pathol 1995;105: infiltra

37 Robbins P, Pinder S, de Klerk N, et al. Histological grading of breast carcinomas. A study of interobserver agreement

38 Sharifi S, Peterson MK, Baum JK, et al. Assessment of prognostic factors in breast core needle biopsies. Mod Pathol 1999;12:941-5.

39 Iczkowski KA, Bostwick DG. The pathologist as optimist: cancer grade deflation in prostatic needle biopsies. $A m{ }^{f}$ Surg Pathol 1998;22:1169-70.

40 Mouriquand J, Gozlan-Fior M, Villemain D, et al. Value of cytoprognostic classification in breast carcinomas. 7 Chin Pathol 1986;39:489-96.

41 van Diest PJ, Mouriquand J, Schipper NW, et al. Prognostic value of nucleolar morphometric variables in cytological breast cancer specimens. F Clin Pathol 1990;43:157-9.

42 Diavan B, Kadesky K, Klopukh B, et al. Gleason scores from prostate biopsies obtained with 18-gauge biopsy needles poorly predict Gleason scores of radical prostatectomy poorly predict Gleason scores of radic

43 Ellis IO, Galea M, Broughton N, et al. Pathological prognostic factors in breast cancer. II. Histological type. Relationship with survival in a large study with long-term follow-up. Histopathology 1992;20:479-89.

44 Dawson PJ, Ferguson DJ, Karrison T. The pathological findings of breast cancer in patients surviving 25 years after radical mastectomy. Cancer 1982;50:2131-8.

45 Dixon JM, Page DL, Anderson TJ, et al. Long-term survivors after breast cancer. Br $\mathcal{F}$ Surg 1985;72:445-8.

46 Pereira H, Pinder SE, Sibbering DM, et al. Pathological prognostic factors in breast cancer. IV: should you be a typer or a grader? A comparative study of two histological prognostic features in operable breast carcinoma. Histopathology 1995;27:219-26.

47 Zakhour H, Wells C, McKee G. Prognostic features and research. In: Diagnostic cytopathology of the breast. London: research. In: Diagnostic cytopathology

48 Carter GL, Allen C, Henson DE. Relation of tumour size, lymph node status, and survival in 24,740 breast cancer lymph node status, and survive

49 Elston CW, Gresham GA, Rao GS, et al. The Cancer Research Campaign (Kings/Cambridge) trial for early breast cancer-pathological aspects. Br f Cancer 1982;45: 655-69.
50 Fisher ER, Sass R, Fisher B, et al. Pathologic findings from the national surgical adjuvant project for breast cancer protocol no 4). Discrimination for tenth year treatment failure. Cancer 1984;53:712-23.

51 Neville AM, Bettelheim R, Gelber RD, et al. Predicting treatment responsiveness and prognosis in node-negative breast cancer. F Clin Oncol 1992;10:696-705.

52 Pain JA, Ebbs S, Henn RPA, et al. Assessment of breast cancer size: a comparison of methods. Eur f Surg Oncol 1992; 18:44-8.

53 Pinder SE, Ellis IO, Galea M, et al. Pathological prognostic factors in breast cancer. III. Vascular invasion: relationship with recurrence and survival in a large study with long-term follow-up. Histopathology 1994;24:41-7.

54 Robertson JFR, Bates K, Pearson D, et al. Comparison of two oestrogen receptor assays in the prediction of the clinical course of patients with advanced breast cancer. $\mathrm{Br} \mathcal{F}$ Cancer 1992;65:727-30.

55 Robertson JFR, Ellis IO, Pearson D, et al. Biological factors of prognostic significance in locally advanced breast cancer. Breast Cancer Res Treat 1994;29:259-64.

56 Blamey RW. Clinical aspects of malignant disease. In: Elston CW, Ellis IO, eds. Systemic pathology. The breast, 3rd ed. London: Churchill Livingstone, 1998:501-13.

57 McCarty Jr KS, Miller LS, Cox EB, et al. Estrogen receptor analyses-correlation of biochemical and immunohistochemical methods using monoclonal antireceptor antibodies. Arch Pathol Lab Med 1985;109:716-21.

58 Barnes DM, Millis RR. Oestrogen receptors: the history, the relevance and the methods of evaluation. In: Kirkham N, Lemoine NR, ed. Progress in pathology, Vol. 2. Edinburgh: Churchill Livingstone 1995:89-114.

59 Ellis IO, Bell J, Todd J, et al. Evaluation of immunoreactivity with antibody NCRC-11 in breast carcinoma. Br f Cancer 1987;56:295-9.

60 Devine PL, McKenzie IFC. Mucins: structure, function, and association with malignancy. Bioessays 1992;14:61925.

61 Poller DN, Hutchings CE, Galea M, et al. p53 protein expression in human breast cancer: relationship to expression of epidermal growth factor, C-erbB-2 protein overexpression, and oestrogen receptor. Br f Cancer 1992;66:583-8.

62 Barbareschi M. Prognostic value of the immunohistochemical expression of $\mathrm{p} 53$ in breast carcinomas. A review of the literature involving over 9000 patients. Applied Immunohistochemistry 1996;4:106-16.

63 Barnes DM, Dublin EA, Fisher CJ, et al. Immunohistochemical detection of $\mathrm{p} 53$ protein in mammary carcinoma: an important new independent indicator of prognosis? Hum Pathol 1993;24:469-76.

64 Lovekin C, Ellis IO, Locker A, et al. C-erbB-2 oncoprotein in primary and advanced breast cancer. Br f Cancer 1991; 63:439-43.

65 Walker RA, Senior PV, Jones JL, et al. An immunohistochemical and in situ hybridisation study of c-myc and c-erbB-2 expression in primary human breast carcinomas. 7 Pathol 1989;158:97-105.

66 Locker AP, Dowle CS, Ellis IO, et al. C-myc oncogene product expression and prognosis in operable breast product expression and prognosis

67 Joensuu H, Toikkanen S, Isola J. Stromal cell cathepsin D expression and long-term survival in breast cancer. $\mathrm{Br} \mathcal{F}$

68 O'Donoghue AE, Poller DN, Bell JA, et al. Cathepsin D in primary breast carcinoma: adverse prognosis is associated with expression of cathepsin D in stromal cells. Breast Cancer Res Treat 1995;22:137-45.

69 Harris AL. What is the biological, prognostic, and therapeutic role of the EGF receptor in human breast cancer? Breast Cancer Res Treat 1994;29:1-2.

70 Pinder S, Wencyk P, Sibbering DM, et al. Assessment of the new proliferation marker MIB1 in breast carcinoma using image analysis: associations with other prognostic factors and survival. Br f Cancer 1995;71:146-9.

71 Gonzalez MA, Pinder SE, Wencyk PM, et al. An immunohistochemical examination of the expression of E-cadherin, $\alpha$ - and $\beta$-catenins and $\alpha 2$ - and $\beta 1$-integrins in invasive breast cancer. F Pathol 1999;187:523-9.

72 Walker RA, Camplejohn RS. DNA Flow cytometry of human breast carcinomas and its relationship to transferrin and epidermal growth factor receptors. F Pathol 1986;150:

73 Dowle CS, Owainati A, Robins A, et al. The prognostic significance of the DNA content of human breast cancer. $\operatorname{BrF}$ Surg 1987;74:133-6. 